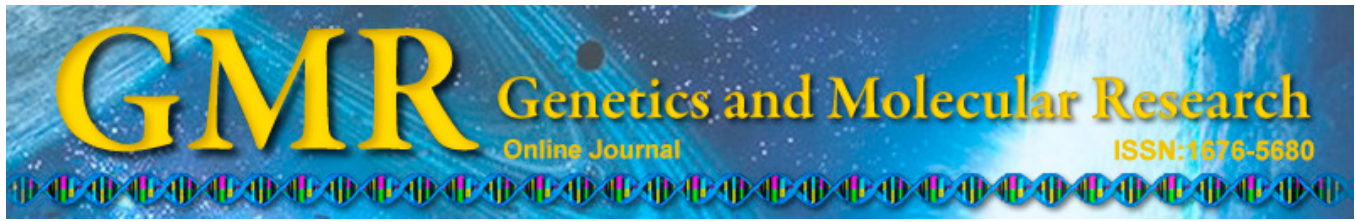

\title{
Genomic selection for slaughter age in pigs using the Cox frailty model
}

\author{
V.S. Santos ${ }^{1}$, S. Martins Filho ${ }^{1}$, M.D.V. Resende ${ }^{2}$, C.F. Azevedo ${ }^{1}$, \\ P.S. Lopes $^{3}$, S.E.F. Guimarães ${ }^{3}$, L.S. Glória ${ }^{3}$ and F.F. Silva ${ }^{3}$ \\ ${ }^{1}$ Departamento de Estatística, Universidade Federal de Viçosa, \\ Viçosa, MG, Brasil \\ ${ }^{2}$ Empresa Brasileira de Pesquisa Agropecuária, Universidade Federal de Viçosa, \\ Centro Nacional de Pesquisa de Florestas, Colombo, PR, Brasil \\ ${ }^{3}$ Departamento de Zootecnia, Universidade Federal de Viçosa, \\ Viçosa, MG, Brasil \\ Corresponding author: V.S. Santos \\ E-mail: 2santosvinicius@gmail.com
}

Genet. Mol. Res. 14 (4): 12616-12627 (2015)

Received May 4, 2015

Accepted August 22, 2015

Published October 19, 2015

DOI http://dx.doi.org/10.4238/2015.October.19.5

\begin{abstract}
The aim of this study was to compare genomic selection methodologies using a linear mixed model and the Cox survival model. We used data from an F2 population of pigs, in which the response variable was the time in days from birth to the culling of the animal and the covariates were 238 markers [237 single nucleotide polymorphism (SNP) plus the halothane gene]. The data were corrected for fixed effects, and the accuracy of the method was determined based on the correlation of the ranks of predicted genomic breeding values (GBVs) in both models with the corrected phenotypic values. The analysis was repeated with a subset of SNP markers with largest absolute effects. The results were in agreement with the GBV prediction and the estimation of marker effects for both models for uncensored data and for normality. However, when considering censored data, the Cox model with a normal random effect (S1) was more appropriate. Since there was no agreement between the linear mixed model and the imputed data
\end{abstract}


(L2) for the prediction of genomic values and the estimation of marker effects, the model S1 was considered superior as it took into account the latent variable and the censored data. Marker selection increased correlations between the ranks of predicted GBVs by the linear and Cox frailty models and the corrected phenotypic values, and 120 markers were required to increase the predictive ability for the characteristic analyzed.

Key words: Censured data; Mixed model; Polymorphism

\section{INTRODUCTION}

Animal breeding programs select individuals that take the shortest possible time to achieve a desirable weight. As some individuals do not reach the desired weight during the study period, models used in survival analysis can be employed, such as the Cox regression model (Giolo et al., 2003).

In genetic studies, individuals of the same family or animals from the same litter have correlated survival times, which violate the assumption of independence between individuals. One way of analyzing such data is by using survival analysis models with correlated frailty (Giolo and Demétrio, 2011). In the context of genome-wide selection (GWS), the correlation matrix associated with frailty is a relationship matrix that is not based on pedigree but on markers, and is known as genomic best linear unbiased prediction (GBLUP) (Meuwissen et al., 2001; VanRaden, 2008).

Several studies have used survival models in animal breeding. In dairy cattle for example, Buenger et al. (2001) and Ojango et al. (2005) assessed the lifetime productivity of cows, and in Nellore beef cattle, Giolo and Demétrio (2011) investigated the number of days required to achieve a standard weight gain. In pig breeding, Mészáros et al. (2010) and Tarres et al. (2006) evaluated the number of days between first farrowing and culling of Large White and Landrace breeds using the Weibull model. However, none of these studies investigated genomic selection.

Previous studies have compared linear models with survival models to obtain information regarding relationships between individual animals. For example, Hou et al. (2009) compared five models, and concluded that the Cox model was the most effective for predicting the breeding values of the traits evaluated. Kärkkäinen and Sillanpaa (2013) studied genomic selection, and compared a linear model with a Bayesian approach via a threshold model proposed for GBLUP, in addition to a multilocus association model with censored data on binary and ordinal scales. The authors concluded that for censored data, the threshold model was more accurate than the linear model, but for binary and ordinal scale data, the superiority of the threshold model has not been confirmed.

GWS studies that have evaluated survival models, such as the Cox model, have not been conducted. Therefore, the main objective of this study was to compare the GBLUP methodology using a traditional linear mixed model and the Cox survival model, which were applied to real data of age at slaughter of an F2 population of pigs (Commercial x Piau). We also evaluated different subsets of markers and selected the one that maximized selection accuracy. 


\section{MATERIAL AND METHODS}

The establishment of the population and the phenotypic measurements were performed at the Pig Breeding Farm of the Department of Animal Science, Federal University of Viçosa, Minas Gerais, Brazil, from November 1998 to July 2001. A population of 345 F2 animals was produced by outbreed crossing two Brazilian native boars (Piau breed) with 18 commercial females (Landrace x Large White x Pietrain) as described by Band et al. (2005).

DNA extraction was performed at the Laboratory of Animal Biotechnology, Department of Animal Science of the Federal University of Viçosa, and genotyping was performed by Golden Gate/VeraCode ${ }^{\circledR}$ technology at the Animal Genetics Laboratory, Embrapa Genetic Resources and Biotechnology, Brasilia, DF, Brazil. The 237 single nucleotide polymorphism markers were distributed on the chromosomes of Sus scrofa as follows: SSC1 (N = 56), SSC4 $(\mathrm{N}=54), \operatorname{SSC} 7(\mathrm{~N}=59), \operatorname{SSC} 8(\mathrm{~N}=31), \operatorname{SSC} 17(\mathrm{~N}=25)$, and SSCX $(\mathrm{N}=12)$.

The desirable weight at slaughter of the animals in this population was around 65 $\mathrm{kg}$ (Band et al., 2005). As was the case in the study by Giolo and Demétrio (2011), the exact time it took an animal to gain the desired weight was not known, as daily weighing was impracticable. Only the ages and weights of the animals at the time of slaughter were known.

Therefore, for the Cox model, censoring was based on the variable live weight at slaughter, i.e., animals that did not reach $65 \mathrm{~kg}$ were referred to as censored (event $=0$ ), whereas animals that reached that weight or more were referred to as a failure (event $=1)$.

The data used in the analysis were corrected for the fixed effects of sex and lot (Silva et al., 2013), and the halothane gene was included as an additional marker. For the prediction of genomic breeding values (GBVs), we used the GBLUP method based on a linear mixed model and on the Cox frailty model. The linear mixed model was as follows:

$$
y=X \beta+Z W m+e
$$

(Equation 1)

where $y$ is the vector of length $n$ of the corrected phenotypes for the fixed effects of sex and lot; $\beta$ is the vector of fixed effects; $g=W m$ is the vector of the individual additive genetic effects (random effects) with mean 0 and variance $W W^{\prime} \sigma_{m}^{2}$, where $W$ is the incidence matrix of markers, $m$ is the vector of random effects of the markers, and $e$ is the vector of random errors normally distributed with mean 0 and variance matrix $I \sigma_{\varepsilon}^{2}$ and $X$ and $Z$ matrices of incidence for $\beta$ and $g$, respectively. The equation of the linear mixed model for the prediction of $g$ using a restricted maximum likelihood (REML)/GBLUP method was as follows:

$$
\left[\begin{array}{cc}
X^{\prime} X & X^{\prime} Z \\
Z^{\prime} X & Z^{\prime} Z+G_{m}^{-1} \frac{\sigma_{\varepsilon}^{2}}{\sigma_{g}^{2}}
\end{array}\right]\left[\begin{array}{l}
\hat{\beta} \\
\hat{g}
\end{array}\right]=\left[\begin{array}{c}
X^{\prime} y \\
Z^{\prime} y
\end{array}\right]
$$

where $G_{m}$ is the genomic relationship matrix, $\sigma_{g}^{2}$ is the variance explained by additive genetic effects, and $\sigma_{\varepsilon}^{2}$ is the residual variance. Once the additive genetic and residual variances were obtained, the heritability calculation was as follows:

$$
h^{2}=\sigma_{g}^{2} /\left(\sigma_{g}^{2}+\sigma_{e}^{2}\right)
$$

(Equation 3) 
VanRaden (2008) demonstrated that the relationship matrix of the marker locus $G_{m}$ is equal to $W W^{\prime} / \sum_{i=1}^{n} 2 p_{i} q_{i}$, where $p_{i}$ is the allele frequency of $\mathrm{M}$ at locus $i$. The incidence matrix $W$ contains wij values of $-1,0$, and 1 for the genotypes of marker types $\mathrm{mm}, \mathrm{Mm}$, and $\mathrm{MM}$, respectively.

According to Resende et al. (2012), the genomic breeding value of individual $j$ in the GBLUP method is given by the following:

$$
\hat{g}=\sum_{i} w_{i j} \hat{m}_{i}
$$

From the estimation of genetic effects $(\hat{g})$ by GBLUP, the estimated effects of the markers $(\hat{m})$ can be obtained by the following:

$$
\hat{g}=W \hat{m} \Rightarrow W^{\prime} \hat{g}=W^{\prime} W \hat{m} \Rightarrow \hat{m}=\left(W^{\prime} W\right)^{-1} W^{\prime} \hat{g} \quad \text { (Equation 5) }
$$

Adopting a form that is similar to the linear mixed model, Therneau (2012) defined the following Cox frailty model, which, in the context of GWS, is given by the following:

$$
h(t)=h_{0}(t) \exp \{X \beta+Z g\}
$$

where $h_{0}(t)$ is an unspecified baseline hazard function, $X$ and $Z$ are the incidence matrices for fixed and random effects, respectively, $\beta$ is the vector of fixed effects, and $g$ is the vector of individual additive genetic effects in the Cox model, which is assumed to be normal, with mean 0 and the following covariance matrix:

$$
\Sigma=G_{m} \sigma_{g}^{2}
$$

As with the linear mixed model, the matrix of variances and covariances was based on the genomic relationship matrix. The matrix $G_{m}$ was computed using the function A.mat $\left(G_{m}\right)$ in the ridge regression-BLUP (rrBLUP) package (Endelman, 2011) in R (R Development Core Team, 2014). To make $G_{m}$ a definite positive matrix, the following matrix, where $I$ is an identity matrix, was obtained:

$$
G_{m}^{*}=G_{m}+10^{-6} I
$$

When $G_{m}^{*}$ was obtained, it was used in the estimators for the calculation of estimates of variance components and random effects for the linear mixed model and the Cox frailty model using the lmekin and coxme functions, respectively, implemented in the coxme package (Therneau, 2012) in R.

Assuming that the random effects or frailties $g$ followed a normal distribution, with mean 0 and the following variance and covariance matrix:

$$
\Sigma=G_{m} \sigma_{g}^{2}
$$

(Equation 9) 
and assuming that censoring is independent and uninformative of $g$, the partial likelihood function for the Cox frailty model is given by the following equation (Pankratz et al., 2005; Giolo and Demétrio, 2011):

$$
L=\int P L(\beta, g) \frac{1}{\sqrt{2 \pi|\Sigma|}} \exp \left[-\frac{1}{2} g^{\prime} \Sigma^{-1} g\right] d g
$$

where $P L$ is the partial likelihood function for the usual Cox model. As the above integral does not provide a closed form, because according to Pankratz et al. (2005), $P L$ is a product of the ratio and the vector of random effects, $g$ has dimension $n$. Ripatti and Palmgren (2000) used the Laplace approximation to obtain the logarithm of the likelihood function (1).

As can be seen, the term referring to the random error is not included in the Cox frailty model, because this term is incorporated into the baseline hazard function (Pankratz et al., 2005). Therefore, heritability cannot be directly calculated. A calculation that is similar to that used in the linear mixed model was initially proposed by Yazdi et al. (2002) and used by Schneider et al. (2005) and Anderson et al. (2007) for the Cox frailty model, which is given by the following equation:

$$
h^{2}=\sigma_{g}^{2} /\left(\sigma_{g}^{2}+\frac{1}{1-c}\right)
$$

where $\sigma_{g}^{2}$ is the variance explained by the additive genetic effects and $c$ is the proportion of censored data. The authors explain that the error variance can be replaced by $1 /(1-c)$. In the case of uncensored data, the error variance becomes 1 , with the random error on the latent scale with a standard normal distribution and a probit link function, as shown by Resende (2002). Mészáros et al. (2010) reported that in some studies involving survival models, heritability is computed assuming that the random error is on the latent scale with an extreme value distribution (Gumbel distribution), according to Resende (2002), with a log-log complement link function and error variance given by $\pi^{2} / 6$.

In order to verify that both linear and Cox models with random effects are consistent in predicting GBVs and in estimating marker effects, the following models were compared: a linear mixed model, which assumed that all observations were uncensored (L1); a linear mixed model with imputed data (L2); a censored Cox frailty model (S1); and an uncensored Cox frailty model (S2).

In the case of incomplete observations, multiple imputations can be performed, in which each missing data is imputed $m$ times, generating $m$ complete databases. Therefore, to compare the models L2 and S1, values of the dependent variable that were considered censored in the model S1 were replaced by the average value of multiple imputations in the model L2. The multiple imputations were performed using the Bayesian linear regression method by the MICE package (Buuren and Groothuis-Oudshoorn, 2011) in R. This method assumes that the multiple imputations are made by means of a multiple linear regression, where the response variable is the variable to be imputed (Nunes et al., 2010).

Cross-validation was performed using the jackknife technique, which involves the 
division of a sample of size $N$ into groups $g$ of equal size to $k$, so that $N=g$, where $k=1$ (Resende, 2002). The procedure was performed as described by Resende et al. $(2010,2012)$, and consisted of deleting an individual analysis for each repetition in order to construct the "validation population"; the other 334 individuals were used to estimate marker effects by the following expression:

$$
\hat{m}=\left(W^{\prime} W\right)^{-1} W^{\prime} \hat{g}
$$

Those individuals constituted the "training population". After obtaining the marker effects, they were applied in the validation population for the prediction of GBVs by the following expression:

$$
\hat{g}=W \hat{m}
$$

After obtaining the predicted GBVs in the cross-validation, they were submitted to correlation analysis with the corrected phenotypic values, in order to assess the predictive ability of the method and the accuracy of the GBV predictions of the models used in an independent sample.

However, a problem arose in obtaining a correlation between the predicted GBVs from the Cox frailty models (S1 and S2) and the phenotype, once the estimated GBVs in those models were represented on a scale of hazard rate and the phenotypes were fixed on a scale of days. To solve this, the correlation between the ranks of GBVs were sorted in increasing order for the linear model (L1 and L2) and in decreasing order for the censored (S1) and uncensored (S2) Cox frailty models, because the hazard rate increased with time. The accuracy of each model, which was the correlation between the ranks divided by the square root of the heritability estimated by maximum likelihood, was also obtained. Pearson and Spearman correlations between the predicted GBVs in the four models were also obtained.

In addition to the GBLUP method, the supervised GBLUP method, which is similar to the RR-BLUP-B method used by Resende Jr. et al. (2012), was also applied. This method firstly predicted GBVs using all of the markers, and then the markers were ranked in descending order based on their estimated effect on the training population and grouped into subsets of markers. A selection of the subset of markers that maximized the predictive ability obtained in the training population was then conducted. Finally, validation was performed in this selected subset, and in all of the smaller subsets and in one larger subset, to ascertain the trend (Resende et al., 2010).

In order to verify the equivalence between models in selecting the best individuals and the largest marker effects, we calculated the concordance rates for the $10 \%$ greatest effects of the predicted GBVs using all 238 markers, and only selected the supervised GBLUP method between similar models L1 and S2 and L2 and S1. The concordance rates for the largest effects in absolute values of all of the markers and those selected in the supervised GBLUP method were also calculated.

In addition to calculating the concordance rate between the models, the kappa $(k)$ index was also obtained, which involved measuring the degree of agreement between the two models in similar situations, i.e., L1 versus S2 and L2 versus S1. The statistical significance of kappa was evaluated using the $\mathrm{Z}$ test, in which the tested hypotheses were the null hypothesis, 
$k=0$ (the agreement between the two methods is null) and the alternative hypothesis, $k>0$ (agreement greater than chance). If the result of the $\mathrm{Z}$ test was greater than the critical value, there was evidence that the classification between the two methods did not occur by chance (Agresti, 2002).

\section{RESULTS}

The correlation values between the ranks of the predicted GBVs and the accuracy of the models obtained by the jackknife cross-validation in the linear and Cox models with different numbers of markers are shown in Table 1. Note that with the full number of markers, none of the evaluated models (L1, L2, S1, or S2) exhibited an adequate predictive ability. However, when using 30 to $50 \%$ of the most significant markers instead of all of them, there was a maximization of the predictive ability of each method, particularly when using the subset of 120 markers that had the largest effects.

Table 1. Predictive ability $\left(r_{y \hat{y}}\right)$ (correlation between the ranks) and accuracy $\left(r_{g \hat{g}}\right)$ of genome-wide selection in the validation population based on linear mixed and Cox frailty models.

\begin{tabular}{|c|c|c|c|c|c|c|c|c|}
\hline \multirow[t]{2}{*}{ Proportion of markers } & \multicolumn{2}{|c|}{$\mathrm{L} 1$} & \multicolumn{2}{|c|}{$\mathrm{L} 2$} & \multicolumn{2}{|c|}{$\mathrm{S} 1$} & \multicolumn{2}{|c|}{ S2 } \\
\hline & $r_{y \hat{y}}$ & $r_{g \hat{g}}$ & $r_{y \hat{y}}$ & $r_{g \hat{g}}$ & $r_{y \hat{y}}$ & $r_{g \hat{g}}$ & $r_{y \hat{y}}$ & $r_{g \hat{g}}$ \\
\hline $30 \%$ (72 markers) & 0.26 & 0.6 & 0.18 & 0.38 & 0.13 & 0.34 & 0.24 & 0.6 \\
\hline 40\% (96 markers) & 0.25 & 0.59 & 0.17 & 0.35 & 0.13 & 0.33 & 0.22 & 0.52 \\
\hline $50 \%$ (120 markers) & 0.22 & 0.55 & 0.16 & 0.33 & 0.12 & 0.32 & 0.21 & 0.49 \\
\hline $75 \%$ (180 markers) & 0.17 & 0.47 & 0.11 & 0.28 & -0.11 & - & -0.12 & - \\
\hline $100 \%$ (238 markers) & 0.08 & 0.28 & 0.03 & 0.13 & 0.01 & 0.04 & -0.02 & - \\
\hline
\end{tabular}

$\mathrm{L} 1$ = linear mixed model with complete data; $\mathrm{L} 2=$ linear mixed model with imputed data; $\mathrm{S} 1$ = censored Cox frailty model; S2 = uncensored Cox frailty model.

In this selected subset, the predictive ability of the models L1 and S2 were almost identical, with values of 0.22 and 0.21 , respectively, indicating that both models, which were similar in their construction, were consistent in predicting the true phenotypic value. For the models L2 and S1, the predictive ability values were very close together, with values of 0.16 and 0.12 , respectively. A similar pattern emerged for the accuracies, with values of 0.33 and 0.32 for the respective models.

In general, we observed a significant increase in heritability estimates of the training population when using a subset of selected markers (Table 2). As was observed for predictive ability and accuracy, the analysis with 50\% (120) of the most significant markers for the four models maximized these estimates, with heritability values of $0.16,0.23,0.14$, and 0.18 for the respective models L1, L2, S1, and S2. These low values are consistent with the nature of this characteristic.

Table 3 presents the estimates of the correlation coefficients of the predicted GBVs between different models under cross-validation, based on the 120 most significant markers. The highest correlations were obtained between the models L1 and S2 (Pearson correlation $=-0.91$ and Spearman correlation $=-0.89$ ), and the lowest between the S1 and L2 models (Pearson correlation $=-0.47$ and Spearman correlation $=-0.44$ ). The correlations between the models L1 and L2 and between S1 and S2 were around 0.5. 
Table 2. Heritability estimated using the genomic best linear unbiased prediction method for linear mixed and Cox frailty models with different numbers of markers.

\begin{tabular}{lcccc}
\hline Proportion of markers & \multicolumn{3}{c}{ Model } \\
\cline { 2 - 5 } & L1 & L2 & S1* & S2* \\
\hline $30 \%$ (72 markers) & 0.19 & 0.22 & 0.15 & 0.16 \\
$40 \%$ (96 markers) & 0.18 & 0.23 & 0.16 & 0.18 \\
$50 \%(120$ markers) & 0.16 & 0.23 & 0.14 & 0.18 \\
$75 \%$ (180 markers) & 0.13 & 0.16 & 0.09 & 0.09 \\
$100 \%$ (238 markers) & 0.08 & 0.05 & 0.02 & 0.02 \\
\hline
\end{tabular}

$\mathrm{L} 1$ = linear mixed model with complete data; $\mathrm{L} 2=$ linear mixed model with imputed data; $\mathrm{S} 1$ = censored Cox frailty model; S2 = uncensored Cox frailty model. *Heritability calculated according to Equation 11.

Table 3. Estimates of the correlation coefficients of the predicted genomic breeding values by different methods under cross-validation, based on the 120 most significant markers using the genomic best linear unbiased prediction method for linear mixed and Cox frailty models.

\begin{tabular}{lcccc}
\hline Model & L1 & L2 & S1 & S2 \\
\hline L1 & - & 0.48 & -0.57 & -0.89 \\
L2 & 0.54 & - & -0.44 & -0.53 \\
S1 & -0.6 & -0.47 & - & 0.52 \\
S2 & -0.91 & -0.57 & 0.54 & - \\
\hline
\end{tabular}

$\mathrm{L} 1=$ linear mixed model with complete data; $\mathrm{L} 2=$ linear mixed model with imputed data; $\mathrm{S} 1=$ censored Cox frailty model; S2 = uncensored Cox frailty model. Correlations between the estimated genomic breeding values in the models; above the diagonal, Spearman's correlation; below the diagonal, Pearson correlation.

The proportion of agreement and the kappa index between the $10 \%$ smallest GBVs $(\widehat{G B V} \bar{V})$ in the linear mixed models (L1 and L2) and the $10 \%$ greatest values $(\bar{G} \vec{B} \bar{V} S)$ in the Cox frailty models (S1 and S2), in addition to the proportion of agreement of the marker effects in different subsets, are shown in Table 4. Based on the most significant 120 markers in the validation population, approximately $71 \%$ of the top 34 individuals (24 animals) were identified in both a linear model (L1) and the uncensored Cox frailty model (S2), with a kappa index value of 0.67, which is classed as in very good agreement (Landis and Koch.1977). However, when evaluating the agreement between the $10 \%$ smallest predicted $(\bar{G} \bar{B} \bar{V} S)$ by the linear mixed model with imputed data (L2) and the $10 \%$ greatest predicted $(\overrightarrow{G B V} S)$ by the censored Cox frailty model (S1), only $35 \%$ of the top 34 individuals (12 animals) were identified in both models, with a kappa index value of 0.28 , which is classed as in reasonably good agreement (Landis and Koch, 1977).

Table 4. Proportion of agreement and kappa index (between parentheses) between methods for the greatest $10 \%$ predicted genomic breeding values $(G B V)$ in the validation population and the loci of the largest effects in the training population for the models $\mathrm{L} 1, \mathrm{~L} 2, \mathrm{~S} 1$, and $\mathrm{S} 2$.

\begin{tabular}{|c|c|c|c|c|}
\hline \multirow[t]{2}{*}{ Proportion of markers } & \multicolumn{2}{|c|}{$\widehat{G B V}$} & \multicolumn{2}{|c|}{ Marker effects } \\
\hline & L1 x S2 & L2 x S1 & L1 x S2 & L2 x S1 \\
\hline $30 \%$ (72 markers) & $0.71(0.67 * *)$ & $0.15\left(0.05^{\mathrm{ns}}\right)$ & $0.78\left(0.68^{* *}\right)$ & $0.49(0.26 * *)$ \\
\hline $40 \%$ (96 markers) & $0.74(0.71 * *)$ & $0.29(0.21 * *)$ & $0.82\left(0.70^{* *}\right)$ & $0.51(0.18 * *)$ \\
\hline $50 \%$ (120 markers) & $0.71(0.67 * *)$ & $0.35(0.28 * *)$ & $0.84\left(0.68^{* *}\right)$ & $0.57\left(0.13^{*}\right)$ \\
\hline $100 \%$ (238 markers) & $0.65(0.61 * *)$ & $0.24(0.15 * *)$ & - & - \\
\hline
\end{tabular}

L1 = linear mixed model with complete data; $\mathrm{L} 2=$ linear mixed model with imputed data; $\mathrm{S} 1=$ censored Cox frailty model; S2 = uncensored Cox frailty model. *Significant at 5\%, **significant at $1 \%$, ${ }^{\mathrm{n}}$ not significant. 
The null hypothesis (that the kappa index was zero) was rejected at a significance level of 1\% for all of the cases evaluated (except for comparisons between models L2 and S1 with 72 markers), indicating that there was a significant correlation in predicted $(\overline{G B V} \bar{S})$ between the models.

For the marker effects, of the 120 markers with the largest absolute effects on the training population, 101 were concordant for both models (L1 and S2), with a percentage of agreement of $84 \%$ and a kappa index value of 0.68 , which is classified as in very good agreement and significant at the 1\% level. When comparing the models L2 and S1, only 68 were concordant for both models, with a percentage of agreement of $57 \%$ and a kappa index value of 0.13 , which is classified as in slight agreement.

\section{DISCUSSION}

This study analyzed the interval in days from birth to slaughter using a linear mixed model and the Cox frailty model in four different scenarios: L1, a linear mixed model that assumed all of the data were uncensored; L2, a linear mixed model with imputed data; S1, a censored Cox frailty model; and S2, an uncensored Cox frailty model. The predictive ability and accuracy of the models were evaluated by the correlation between the predicted GBVs in the cross-validation and the phenotypic values corrected for their fixed effects. In addition, we evaluated several subsets of markers in order to select the one that maximized the predictive ability in the training population.

The correlations between the ranks, and consequently the accuracies, were higher in models L1 and S2 than in models L2 and S1. This was because the models L1 and S2 did not consider the nature of the censoring, and the censored times were considered as exact times. As to the models L2 and S1, the distance between the predicted GBVs and the corrected phenotypic values of the censored time was expected to be higher. Although the accuracies estimated in the models L1 and S2 were higher, this did not mean that these models were the best, since the phenotypic values used in the validation were discreet and were not expressed on the latent scale, which was the most appropriate. The models L2 and S1 were conceptually more complete, since they expressed the correlations with the phenotypic values on the latent scale.

The analysis with 50\% (120) of the markers with the largest effects for the four models maximized the predictive ability and the heritability. This marker selection strategy was also applied by Resende Jr. et al. (2012) in pine (Pinus taeda L.) using the RR-BLUP method, which the authors termed RR-BLUP-B. The method's performance was better than that of RR-BLUP, and similar to Bayesian methods for the characteristics of rust disease resistance and wood density. The advantage of this method, according to Resende Jr. et al. (2012), is its simplicity and lower computational demand than Bayesian methods.

The heritability estimates found in this study using the L1 and L2 models based on 120 markers with the largest effects agree with those obtained by Torres Filho et al. (2004), who reported values of between 0.13 and 0.20 for age until achieving $100 \mathrm{~kg}$ in Large White pigs. For the models S1 and S2, the heritability was calculated using Equation 11, with the error variance replaced by $1 /(1-c)$, where $c$ is the proportion of censored data. For the censored Cox frailty model (S1), the value of $c$ was 0.56 , and for $\mathrm{S} 2$, the error variance was 1 , because the random error on the latent scale was modeled assuming a standard normal distribution with a probit link function. In proportional hazard models, such as the Weibull and Cox models, the 
random error component is incorporated into the baseline hazard function, so it is not possible to directly obtain an estimated heritability.

According to Pankratz et al. (2005) and Giolo and Demétrio (2011), another way to obtain information about heritability is by using a measure of risk, since the variance components obtained in the Cox frailty model are modeled on the log-hazard scale. In other words, the value obtained by the exponential of the square root from the genetic variance provides information about the relative risk associated with the random effects.

The estimates of the components of polygenic variance were 0.38 and 0.22 in the censored and uncensored Cox models, respectively (results not shown). The risk of an animal achieving slaughter weight gain in a shorter period was around $\exp (\sqrt{ } 0.38) \approx 1.852$ for the censored Cox frailty model and $\exp (\sqrt{ } 0.22) \approx 1.598$ for the uncensored Cox frailty model. According to Pankratz et al. (2005) and Giolo and Demétrio (2011), there were animals with a $85 \%$ greater risk of obtaining the ideal slaughter weight than the overall average risk based on the censored Cox frailty model, and a $60 \%$ greater risk based on the uncensored Cox frailty model.

When the most informative markers (120 markers) were used, the heritability of 0.02 was increased to 0.14 and 0.18 for the censored (S1) and uncensored (S2) Cox models, respectively. Mészáros et al. (2010) evaluated the number of days between first farrowing and culling of Large White and Landrace pigs using the Weibull survival model, and found heritability values of 0.08 and 0.05 for the respective breeds based on an animal model. The authors used the same expression as used in this study and proposed by Yazdi et al. (2002) for the calculation of heritability. Analyzing the same variable in the same breeds, Serenius and Stalder (2004) found heritabilities of 0.16 and 0.17 for Landrace and 0.17 to 0.19 for Large White pigs. Yazdi et al. (2000) reported heritability values for longevity in Landrace pigs that ranged from 0.11 to 0.27 .

According to Mészáros et al. (2010), in these last two studies, heritability estimates were obtained assuming that the random error on the latent scale exhibited a Gumbel or extreme value distribution, with a log-log complement link function and a variance $\pi^{2} / 6$. In this case, heritability is defined on the logarithmic scale, and not on the original scale as proposed by Yazdi et al. (2002); therefore, a direct comparison is not possible.

Therefore, Mészáros et al. (2010) suggested replacing the variance components in Equation 11 with those obtained according to the model used, in order to make comparable estimates of heritability. By doing so, the new value of heritability obtained, based on Yazdi et al. (2000), was 0.09, and in the study by Serenius and Stalder (2004) it ranged between 0.18 and 0.21 , which was close to that obtained by the same authors ( 0.16 to 0.19$)$.

More accurate studies on heritability that have evaluated age from birth to culling were not found in the literature, with the above mentioned variable being the closest for comparison; moreover, all of the heritability values that were found in the literature were based on a relationship matrix that was associated with pedigree. It is also worth noting that, when comparing these heritabilities, the survival model and the covariates used should be taken into account, in addition to the expression used for the calculation of heritability (Mészáros et al., 2010).

A negative correlation was observed between the predicted $\widehat{G B V} S$ in the linear models (L1 and L2) and the censored (S1) and uncensored (S2) Cox models. This was because the time variable was directly modeled in the linear model, which in this study was the time to reach the optimal slaughter weight; however, in the Cox model it has been modeled as the risk 
of reaching the ideal slaughter weight (Hou et al., 2009; Ellen et al., 2010).

The model L1 exhibited a correlation of around $60 \%$ with the model S1, which was the most suitable value, while for the models L2 and S2 the correlation was around 50\%. Therefore, there was a large difference between S1 and the other models, which reflects the improvement of using the censored Cox model. Hou et al. (2009) evaluated the interval between calving and first insemination and between calving and last insemination in dairy cattle, and concluded that among the evaluated models, the Cox model was the best in predicting the breeding values of the two traits.

In the models L1 and L2, the best animals were those with small $(\widehat{G B V} S)$ once they had reached the desired slaughter weight in less time. Regarding models S1 and S2, the best animals were those that had high $(\overrightarrow{G B V S})$, as the random effect was frailty; therefore, the hazard function rapidly increased, indicating that the animal's weight also rapidly increased (Giolo et al., 2003; Hou et al., 2009).

When comparing the models L1 and S2, the proposed method using the genomic relationship matrix based on the Cox model with normal random effects was satisfactory, because it selected about $71 \%$ of the same individuals and $84 \%$ (training population) of the same markers when using the linear mixed model in situations of normally distributed data, $0 \%$ censoring, and selection of the 120 most significant markers.

However, assuming model S1 as true, the linear model with imputation for censored values in the model S1 was not efficient in selecting the best individuals and markers with the largest effects when compared to the Cox frailty model in the case of censoring (S1) and selection of the 120 most significant markers, because it selected only $35 \%$ of the same individuals and about $57 \%$ of the same markers.

In summary, in the case of normality and uncensored data, the Cox model with normally distributed random effects (S2) and the linear mixed model (L1) produced concordant results in the prediction of GBVs and the estimation of marker effects. However, in the case of censoring these results were discordant, indicating that the Cox model with normal random effects (S1) is the most appropriate in these situations, because it takes into account latent variables and censoring in the data.

\section{Conflicts of interest}

The authors declare no conflict of interest.

\section{ACKNOWLEDGMENTS}

V.S. Santos would like to thank Fundação de Amparo à Pesquisa do Estado de Minas Gerais (FAPEMIG) for a scholarship. Research supported by Conselho Nacional de Desenvolvimento Científico e Tecnológico (CNPq) and Fundação de Amparo à Pesquisa do Estado de Minas Gerais (FAPEMIG).

\section{REFERENCES}

Agresti A (2002). Categorical data analysis. John Wiley and Sons, New Jersey.

Anderson CA, Duffy DL, Martin NG and Visscher PM (2007). Estimation of variance components for age at menarche in twin families. Behav. Genet. 37: 668-677.

Band GO, Guimarães SEF, Lopes PS, Peixoto JDO, et al. (2005). Relationship between the Porcine Stress Syndrome gene 
and carcass and performance traits in F2 pigs resulting from divergent crosses. Genet. Mol. Biol. 28: 92-96.

Buenger A, Ducrocq V and Swalve HH (2001). Analysis of survival in dairy cows with supplementary data on type scores and housing systems from a region of northwest Germany. J. Dairy Sci. 84: 1531-1541.

Buuren S and Groothuis-Oudshoorn K (2011). MICE: Multivariate Imputation by Chained Equations in R. J. Stat. Soft. 45: 1-67.

Ellen ED, Ducrocq V, Ducro BJ, Veerkamp RF, et al. (2010). Genetic parameters for social effects on survival in cannibalistic layers: combining survival analysis and a linear animal model. Genet. Sel. Evol. $42: 27$.

Endelman JB (2011). Ridge regression and other kernels for genomic selection with R package rrBLUP. Plant Gen. 4: 250-255.

Giolo SR and Demétrio CGB (2011). A frailty modeling approach for parental effects in animal breeding. J. Appl. Stat. 38: 619-629.

Giolo SR, Henderson R and Demétrio CGB (2003). Um critério para seleção de touros Nelore usando modelos de sobrevivência. Rev. Bras. Biom. 21: 115-123.

Hou Y, Madsen P, Labouriau R, Zhang Y, et al. (2009). Genetic analysis of days from calving to first insemination and days open in Danish Holsteins using different models and censoring scenarios. J. Dairy Sci. 92: 1229-39.

Kärkkäinen HP and Sillanpaa MJ (2013). Fast genomic predictions via Bayesian G-BLUP and multilocus models of threshold traits including censored Gaussian data. G3 3: 1511-1523.

Landis JR and Koch GG (1977). The measurement of observer agreement for categorical data. Biometrics 33: 159-174.

Mészáros G, Palos J, Ducrocq V and Solkner J (2010). Heritability of longevity in Large White and Landrace sows using continuous time and grouped data models. Genet. Sel. Evol. 42: 13.

Meuwissen TH, Hayes BJ and Goddard ME (2001). Prediction of total genetic value using genome-wide dense marker maps. Genetics 157: 1819-1829.

Nunes LN, Kluck MM and Fachel JM (2010). Comparison of simple and multiple imputation methods using a risk model for surgical mortality as example. Rev. Bras. Epidemiol. 13: 596-606.

Ojango JMK, Ducrocq V and Pollott GE (2005). Survival analysis of factors affecting culling early in the productive life of Holstein-Friesian cattle in Kenya. Livest. Prod. Sci. 92: 317-322.

Pankratz VS, Andrade M and Therneau TM (2005). Random-effects Cox proportional hazards model: general variance components methods for time-to-event data. Genet. Epidemiol. 28: 97-109.

R Development Core Team (2014). R: A Language and Environment for Statistical Computing. Available at [http:// www.R-project.org]. Accessed August 20, 2014.

Resende MDV (2002). Genética biométrica e estatística no melhoramento de plantas perenes. Embrapa Florestas, Colombo.

Resende MDV, Resende Jr MFR, Aguiar AM, Abad JIM, et al. (2010). Computação da seleção genômica ampla (GWS). Embrapa Florestas, Colombo.

Resende MDV, Silva FF, Lopes PS and Azevedo CF (2012). Seleção genômica ampla (GWS) via modelos mistos (REML/ BLUP), inferência Bayesiana (MCMC), regressão aleatória multivariada (RRM) e estatística espacial. Universidade Federal de Viçosa/Departamento de Estatística, Viçosa.

Resende Jr MFR, Munoz P, Resende MDV, Garrick DJ, et al. (2012). Accuracy of genomic selection methods in a standard data set of loblolly pine (Pinus taeda L.). Genetics 190: 1503-1510.

Ripatti S and Palmgren J (2000). Estimation of multivariate frailty models using penalized partial likelihood. Biometrics 56: 1016-1022.

Schneider MDP, Strandberg E, Ducrocq V and Roth A (2005). Survival analysis applied to genetic evaluation for female fertility in dairy cattle. J. Dairy Sci. 88: 2253-2259.

Serenius T and Stalder KJ (2004). Genetics of length of productive life and lifetime prolificacy in the Finnish Landrace and Large White pig populations. J. Anim. Sci. 82: 3111-3117.

Silva FF, Resende MDV, Rocha GS, Duarte DA, et al. (2013). Genomic growth curves of an outbred pig population. Genet. Mol. Biol. 36: 520-527.

Tarres J, Bidanel JP, Hofer A and Ducrocq V (2006). Analysis of longevity and exterior traits on Large White sows in Switzerland. J. Anim. Sci. 84: 2914-2924.

Therneau T (2012). Mixed effects Cox models. R package version 2.2-3. Available at [http://cran.r-project.org/web/ packages/coxme/vignettes/coxme.pdf]. Accessed January 7, 2014.

Torres Filho RA, Torres RA, Lopes PS, Euclydes RF, et al. (2004). Avaliação de modelos para estimação de componentes de (co)variância em características de desempenho e reprodutivas em suínos. R. Bras. Zootec. 33: 350-357.

VanRaden PM (2008). Efficient methods to compute genomic predictions. J. Dairy Sci. 91: 4414-4423.

Yazdi MH, Rydhmer L, Ringmar-Cederberg E, Lundeheim N, et al. (2000). Genetic study of longevity in Swedish Landrace sows. Livest. Prod. Sci. 63: 255-264.

Yazdi MH, Visscher PM, Ducrocq V and Thompson R (2002). Heritability, reliability of genetic evaluations and response to selection in proportional hazard models. J. Dairy Sci. 85: 1563-1577. 